combination of both conditions. ${ }^{2}$ For that reason the authors of the tool have recommended it be used sequentially to identify decline over time from an individual baseline. It cannot be used as a cross-sectional staging tool because a high score could indicate a long-standing level of intellectual ability rather than dementia and a low score might not exclude dementia in those with mild intellectual impairment. Unfortunately clinician ratings of mild, moderate or severe dementia were incompletely recorded in clinical notes (Table 1) and these data were not recorded beyond baseline, thus could not be included in our analyses.

However, in order to examine change in cognitive and functional ability over time from a baseline, our analysis of DLD scores was conducted using coefficients (i.e. based upon the mean difference between the scores of those on medication and those not on medication) that did take account of baseline DLD scores (Table 3). As requested by Professor Buckley, we now report raw DLD data at baseline, and follow-up visits for all individuals for whom this is available. Mean baseline DLD cognitive score in the untreated group was 30.54 (95\% CI 26.49-34.60) and in the treated group 25.35 (95\% CI 23.2927.41); at first follow-up assessment DLD cognitive scores were 27.80 (95\% CI 23.24-32.35) in the untreated group and 22.34 (95\% CI 20.16-24.52) in the treated group; at second follow-up 31.62 (95\% CI 26.17-27.08) (untreated) and 23.90 (95\% CI 21.85-25.88) (treated); and at third follow-up 34.86 (95\% CI 27.49-42.23) (untreated) and 26.20 (95\% CI 23.90-28.51) (treated). These unadjusted data highlight the difference between the group means in cognitive score at baseline and other time points and appear to demonstrate a generally slower rate of cognitive decline in people prescribed medication, with DLD cognitive scores of those not treated worsening by approximately $14 \%$ (increase in scores of 4.32 on average, from a baseline of 30.54) by the third follow-up visit, compared with a worsening in DLD cognitive score of only $3 \%$ on average (increase in scores of 0.85 on average) in those prescribed medication. We have included the third time point here, which we did not include in the analysis in the paper, although as indicated by the width of the confidence interval, the number of observations at this time point is small, particularly in the untreated group. The numbers included at each time point are slightly different from those reported in the paper because of missing data in certain individuals precluding adjustment by baseline value. While we agree that research abstracts are limited by word counts, we believe our reporting is balanced and fair and call for more research in this field, including clinical trials of medication where the limitations of observational designs could be overcome.

1 National Institute for Health and Care Excellence. Dementia: Supporting People with Dementia and their Carers in Health and Social Care. CG42. NICE, 2006.

2 Eurlings HAL, Evenhuis HM, Kengen MMF. Dementia Questionnaire for People with Learning Disabilities (DLD). UK Adaptation. Harcourt Assessment, 2006.

Rory Sheehan, on behalf of all the authors of the article; Nicole Eady, Division of Psychiatry, University College London, UK; Rory Sheehan, Division of Psychiatry, Psychiatry, University College London, UK; Rory Sheehan, Division of Psychiatry,
University College London, UK; Khadija Rantell, Education Unit, Institute of Neurolog, UK; Amanda Sinai, Division of Psychiatry, University College London, UK; Jane Bernal Cornwall Partnership Foundation Trust, UK; Ingrid Bohnen, Westminster Learning Disability Partnership, UK; Simon Bonell, Plymouth Community Learning Disabilities Team, Livewell Southwest, UK; Ken Courtenay, Haringey Learning Disability Partnership, Barnet Enfield Haringey Mental Health NHS Trust, UK; Karen Dodd, Surrey and Borders Partnership NHS Foundation Trust, UK; Dina Gazizova, Hillingdon Learning and Borders Partnership NHS Foundation Trust, UK; Dina Gazizova, Hillingdon Learning
Disabilities Service, UK; Angela Hassiotis, Division of Psychiatry, University College London, UK; Richard Hillier, Islington Learning Disabilities Partnership, UK; Judith McBrien, Plymouth Teaching Primary Care Trust, UK; Kamalika Mukherji, Hertfordshire Partnership NHS Foundation Trust, UK; Asim Naeem, South West London and St George's Mental Health NHS Trust, UK; Natalia Perez-Achiaga, Royal Borough of Kensington and Chelsea Learning Disability Service, UK; Vijaya Sharma, Hertfordshire Partnership NHS Foundation Trust, UK; David Thomas, Hackney Learning Disability Team, East London NHS Foundation Trust, UK; Zuzana Walker, Division of Psychiatry, University College London, UK; Jane McCarthy, Institute of Psychiatry, Psychology and Neuroscience, King's College London, UK; André Strydom, Division of Psychiatry,
University College London, Institute of Psychiatry, Psychology and Neuroscience, King's college London and The LonDowns Consortium, UK. Email: r.sheehan@ucl.ac.uk

doi:10.1192/bjp.2018.194

\section{Difficulties of diagnosing and managing dementia in people with Down syndrome}

As practising clinicians who provide care to adults with Down syndrome, we appreciate Eady and colleagues' attempt to address a real concern in this patient population. ${ }^{1}$ However, we have significant concerns about clinicians potentially using in their practice the conclusions drawn by the authors. The study conclusions offer false hope and may result in wasted resources.

The authors themselves state that the treated and untreated groups have significant differences that would favour the treated group: 'There were significant baseline differences between the groups prescribed and not prescribed antidementia medication. Those who were not prescribed medication were older, more likely to have severe-profound intellectual disability, and had more severe dementia symptoms at baseline'. Given those differences, it is difficult to understand how the authors can come to the conclusion that treatment with antidementia medications is of benefit.

Also of concern is the question of what the clinical significance would be from a functional perspective. Dementia takes a tremendous toll on the caregivers and families. Even if the medications do extend life, where is the benefit? What kind of life will they have? We believe quality of life would have been a more useful measured outcome.

Furthermore, there are four published studies and Cochrane reviews that show no benefit with donepezil, ${ }^{2}$ rivastigmine, ${ }^{3}$ memantine $^{4,5}$ or galantamine. ${ }^{6}$ Another Cochrane Review in 2015 showed no benefit of pharmacological interventions for cognitive decline in people with Down syndrome. ${ }^{7}$

In our experience as the directors of Down syndrome clinics for adults, the big issue is really how the diagnosis of dementia is made. Clinicians tend to easily apply the diagnosis of Alzheimer's dementia without looking at all the potential causes of pseudodementia in this population. ${ }^{8}$ They often assume that loss of ability is as a result of dementia because of a study published in 1985 that showed plaques and tangles in the brain tissue of all people with Down syndrome over the age of $35 .{ }^{9}$ Wisniewski \& Rabe subsequently wrote that there was a discrepancy between neuropathology and the occurrence of dementia in people with Down syndrome. ${ }^{10}$ Just as in the population of typically developed older adults, the diagnosis of Alzheimer's dementia in people with Down syndrome should be made only after evaluation for causes of pseudodementia.

1 Eady N, Sheehan R, Rantell K, Sinai A, Bernal J, Bohnen I, et al. Impact of cholinesterase inhibitors or memantine on survival in adults with Down syndrome and dementia: clinical cohort study. Br J Psychiatry 2018; 212: 155-60.

2 Mohan M, Carpenter PK, Bennett C. Donepezil for dementia in people with Down syndrome. Cochrane Database Syst Rev 2009; 1: CD007178.

3 Mohan M, Bennett C, Carpenter PK. Rivastigmine for dementia in people with Down syndrome. Cochrane Database Syst Rev 2009; 1: CD007658.

4 McShane R, Areosa Sastre A, Minakaran N. Memantine for dementia. Cochrane Database Syst Rev 2006; 2: CD003154.

5 Hanney M, Prasher V, Williams N, Jones EL, Aarsland D, Corbett A, et al. Memantine for dementia in adults older than 40 years with Down's syndrome (MEADOWS): a randomised, double-blind, placebo-controlled trial. Lancet 2012; 379: 528-36.

6 Mohan M, Bennett C, Carpenter PK. Galantamine for dementia in people with Down syndrome. Cochrane Database Syst Rev 2009; 1: CD007656.

7 Livingstone N, Hanratty J, McShane R, Macdonald G. Pharmacological interventions for cognitive decline in people with Down syndrome. Cochrane Database Syst Rev 2015; 10: CD011546. 
8 Chicoine B, McGuire D, Hebein S, Gilly D. Development of a clinic for adults with Down syndrome. Ment Retard 1994; 32: 100-6.

9 Wisniewski KE, Wisniewski HM, Wen GY. Occurrence of neuropathological changes and dementia of Alzheimer's disease in Down's syndrome. Ann Neurol 1985; 17: 278-82.

10 Wisniewski HM, Rabe A. Discrepancy between Alzheimer-type neuropathology and dementia in persons with Down's syndrome. Ann N Y Acad Sci 1986; 477: 247-60.

David Smith, Physician, Down Syndrome Clinic of Wisconsin, USA; Brian Chicoine Advocate Medical Group Adult Down Syndrome Center, USA. Email: dssmith@mac.com doi:10.1192/bjp.2018.199

\section{Author's reply to: Difficulties of diagnosing and managing dementia in people with Down syndrome}

We thank Drs Smith and Chicoine for their interest in our work and highlighting some of the practical issues in diagnosing and managing dementia in this group. The association between trisomy 21 and early-onset Alzheimer's disease is well established ${ }^{1}$ and dementia is now the most common cause of death in adults with Down syndrome. Despite this, there exists relatively little evidence on which to base treatment decisions.

Using a naturalistic study design, we report the effects of antidementia medication on the survival and function of 310 people with Down syndrome and dementia. Notwithstanding the limitations typical of observational studies (discussed in the paper), this work addresses a significant gap in the literature. Kaplan-Meier survival curves demonstrate significantly increased survival in the group prescribed antidementia medication. Baseline differences between those prescribed and not prescribed antidementia medication were accounted for, where possible, in a Cox regression model. This adjusted analysis showed that protection in the treated group remained, although it did not reach statistical significance because of less power and broader confidence intervals.

Functional impairment was measured using the Dementia in Learning Disabilities scale, ${ }^{2}$ a standardised informant questionnaire that covers several skill domains. These data show an early protective effect of medication in mitigating cognitive decline, as is observed in individuals with Alzheimer's disease without Down syndrome. ${ }^{3}$ We appreciate the concern of Drs Smith and Chicoine for quality of life. Unfortunately, there are no well-validated measures of quality of life for this group and proxy measures have been subject to limitations in people with intellectual disability. Development of such measures and their use in research studies and routine clinical care would be welcome and could focus efforts on providing optimal holistic support.

The Cochrane reviews that Drs Smith and Chicoine cite highlight the lack of evidence in this field, rather than negative results of drug intervention studies. Two of these Cochrane reviews did not include any studies at all, and the third included only one, small randomised controlled trial. The authors of these reviews, now some years old, highlight the paucity of evidence and conclude that the reviews cannot be used to guide practice.

Our cohort was recruited from specialist memory clinics for people with intellectual disability. Clinician diagnosis of dementia in such clinics is valid and reliable ${ }^{4}$ and we are confident that clinicians will have adequately assessed potentially reversible causes of decline. It is important not to overlook dementia as an early diagnosis can facilitate prompt pharmacological and psychosocial treatments and effective care planning. ${ }^{5}$ When dementia is diagnosed, a decision to use medication is, of course, an individual one, and should take account of the views of families and carers. Our paper provides additional evidence that could inform the decision- making process. People with Down syndrome and dementia should not be denied access to antidementia drugs.

1 Zis $P$, Strydom A. Clinical aspects and biomarkers of Alzheimer's disease in Down syndrome. Free Radic Biol Med 2018; 114: 3-9.

2 Eurlings H, Evenhuis HM, Kengen M. Dementia Questionnaire for People with Learning Disabilities (DLD). UK Adaptation. Harcourt Assessment, 2006.

3 Perera G, Khondoker M, Broadbent M, Breen G, Stewart R. Factors associated with response to acetylcholinesterase inhibition in dementia: a cohort study from a secondary mental health care case register in London. PLOS One 2014; 9: e109484.

4 Sheehan R, Sinai A, Bass N, Blatchford P, Bohnen I, Bonell S, et al. Dementia diagnostic criteria in Down syndrome. Int J Geriatr Psychiatry 2015; 30: 857-63.

5 Dodd K, Watchman K, Janicki MP, Coppus A, Gaertner C, Fortea J, et al Consensus statement of the international summit on intellectual disability and dementia related to post-diagnostic support. Aging Ment Health 2017; Sept 7 (Epub ahead of print).

Nicole Eady, Division of Psychiatry, University College London, UK: Rory Sheehan Division of Psychiatry, University College London, UK; Khadija Rantell, Education Unit, Institute of Neurology, UK; Amanda Sinai, Division of Psychiatry, University College London, UK; Jane Bernal, Cornwall Partnership Foundation Trust, UK; Ingrid Bohnen, Westminster Learning Disability Partnership, UK; Simon Bonell, Plymouth Community Learning Disabilities Team, Livewell Southwest, UK; Ken Courtenay, Haringey Learning Disability Partnership, Barnet Enfield Haringey Mental Health NHS Trust, UK:

Karen Dodd, Surrey and Borders Partnership NHS Foundation Trust, UK:

Dina Gazizova, Hillingdon Learning Disabilities Service, UK; Angela Hassiotis, Division of Psychiatry, University College London, UK; Richard Hillier, Islington Learning Disabilities Partnership, UK; Judith McBrien, Plymouth Teaching Primary Care Trust, UK; Kamalika Mukherji, Hertfordshire Partnership NHS Foundation Trust, UK;

Asim Naeem, South West London and St George's Mental Health NHS Trust, UK:

Natalia Perez-Achiaga, Royal Borough of Kensington and Chelsea Learning Disability

Service, UK; Vijaya Sharma, Hertfordshire Partnership NHS Foundation Trust, UK;

David Thomas, Hackney Learning Disability Team, East London NHS Foundation Trust, UK; Zuzana Walker, Division of Psychiatry, University College London, UK;

Jane McCarthy, Institute of Psychiatry, Psychology and Neuroscience, King's College London, UK; André Strydom, Division of Psychiatry, University College London, Institute of Psychiatry, Psychology and Neuroscience, King's College London and The LonDowns Consortium, UK. Email: r.sheehan@ucl.ac.uk

doi:10.1192/bjp.2018.208

\section{An alternative perspective on cooper et al's finding of a high incidence of mania in individuals with intellectual disabilities}

Cooper et al (2018) note that despite high use of mood stabilisers (22.4\%), the 2-year incidence of mania in individuals with intellectual disabilities is $1.1 \% .{ }^{1}$ This is higher than in the general population. They infer that clinicians need to consider mania in their differential diagnosis, highlighting the risk of misdiagnosis. The authors specifically note the similarity of symptoms across diagnostic categories, including those for mania, attention-deficit hyperactivity disorder (ADHD) and problem behaviours.

This raises an interesting point of symptom overlap between $\mathrm{ADHD}$ and bipolar disorder, which, as the authors suggest, can lead to diagnostic overshadowing. However, it is possible that this overlap could result in clinicians primarily diagnosing bipolar disorder, with ADHD remaining undiagnosed. This alternative perspective could offer an explanation for the high incidence of mania in the context of high mood stabiliser use.

In Cooper et al's (2007) original study, it is of interest that there were no individuals with ADHD identified within the cohort of mild intellectual disability. ${ }^{2}$ Although the authors acknowledged they might not have fully identified this group, the finding is noteworthy given the average prevalence in the general population is $3.4 \%$ (range 1.2-7.3\%). ${ }^{3}$ This baseline comparative data used in Cooper et al's (2018) report underlines their comments pertaining to the diagnostic challenge of mental illness in the population with intellectual disabilities. ${ }^{1}$

Overall, Cooper et al's recent paper highlights a need for clinicians to be more aware of symptom overlap in the area of 